\title{
Unit management and the role of doctors in local decision making
}

\author{
WILFRED J DAVEY
}

The 1982 reorganisation of the National Health Service was welcomed by most clinicians, who applauded the aim of bringing decision making closer to the operational level. There was to be maximum delegation to units of management, with substantial responsibilities vested in a unit administrator and director of nursing services who were to discharge their responsibilities in conjunction with a senior member of the medical staff. At this time of renewed upheaval, with the imminent appointment of unit general managers, the reaction of unit medical representatives to these arrangements and the demands that have been placed on them is particularly apposite.

Since October 1983 a small team of researchers at the Health Services Management Centre of Birmingham University has been studying the work of the unit management groups. The aims were to observe and describe management activity at unit level, including the organisational arrangements within which unit management groups work and their participation in the monitoring, control, and planning processes; to assess their influence on health service performance; and to cooperate with health authorities in finding ways to improve management effectiveness at the unit level.

Twenty unit management groups and their authorities have cooperated in the main study, in which the researchers have interviewed unit management group members and others with an interest in, or influence on, unit management. We have attended unit management group meetings as observers and studied their minutes and agendas. The experience reported by unit medical representatives and the views of other doctors concerned in the medical representative system have made an important contribution to our findings.

\section{How does a doctor become a unit medical representative?}

Our research showed that unit medical representatives have been chosen in one of three ways. Firstly, the additional responsibility of being unit medical representative might be conferred on a doctor already holding a chairmanship, probably of a clinical division or of a hospital staff committee. This dual role, though burdensome, was thought to function more smoothly than a separation of the two functions, on the one hand coordinating the opinions among clinicians and on the other hand liaising with the unit administrator and director of nursing services. Secondly, in some cases, the choice was a narrow one. One example was of a mental handicap unit in which only one doctor was employed in the specialty. In other single specialty units the number of consultants was frequently small enough for the appropriateness of one person to be self evident. The third kind of selection arrangement occurred where the role of the unit medical representative was seen as relatively unimportant by clinicians or where no clearly eligible candidate could be identified

West Midlands Regional Health Authority, Birmingham B15 1SW WILFRED J DAVEY, MSC, AHSM, senior principal training officer

Correspondence to: West Midlands Regional Health Authority, 1st Floor, Cumberland House, 200 Broad Street, Birmingham B15 ISW. from a considerable list of possibles. It might almost be called selection by default. As one consultant surgeon who found himself nominated at a meeting of the medical advisory committee put it, "People were a bit worried about what would be entailed. I said I thought we could do this with a short period each week providing the necessary advice ... and so they said, 'Right, you're it.',"

There was a predictable consistency in the way in which the unit medical representatives described the time committed to unit work. Attending unit management group meetings was the primary obligation and in all cases this was undertaken with diligence. Many unit management groups found that other demands obliged them to arrive late for meetings or to leave early, and agenda items were sometimes rearranged to fit in with the time that could be spared. Some unit management groups did not accept deputies and if the unit management representative was away no formal meeting would be held. Even where deputies were identified they were not much used and it was felt that continuity over a series of meetings made it difficult for any temporary attender to make the full contribution of the regular clinician. Unit management groups who had more than one doctor among their membership found that to some degree each doctor could cover for the other's occasional absence.

Attempts to define the amount of time that unit medical representatives committed to unit management group business were inhibited by the imprecise boundary between unit management group related activity and that which might arise from participation with other committees. Of the 21 unit medical representatives from whom an estimation of time spent was obtained, five reported spending one day a week or more on unit business, six at least half a day but less than one full day, five at least two and a half hours a week but less than half a day, and five less than two and a half hours a week. These are average figures and include attendance at unit management group meetings, informal discussion with clinical colleagues-for example, over the telephone or at lunch in the postgraduate medical centre-and reading papers and reports. Only one consultant, in a medium sized acute unit, said that he regularly committed ideas to paper as a means of sounding out clinical colleagues and putting proposals to his unit management group. The lowest figures came from geographically dispersed community units where there was relatively little managerial activity for the unit as a whole.

\section{How unit medical representatives see their contribution}

At unit management group meetings the unit medical representatives saw themselves as providing a medical opinion and defending the interests of clinical services by ensuring that administrative decisions did not adversely affect patient care. They all saw themselves as contributing as equal members of the unit group in the sense that they offered views freely on all matters that came forward for discussion whether or not they had a specific clinical content. Many doctors expressed severe doubts as to the extent to which they could properly "represent" their colleagues. Certainly they felt able to express views from a medical standpoint and they were able to indicate in a general way what their colleagues thought on any matter and would find acceptable. It remains in the nature of the autonomy that clinicians cherish that one of their number 
cannot commit his fellows without prior reference to them. The dilemma was particularly strong for general practitioners on unit management groups since these doctors lack the opportunity of any regular contact with more than a small proportion of all the general practitioners within their unit. Most general practitioners see the family practitioner committee and local medical committee as more appropriate mechanisms for voicing opinion. In fact general practitioners on unit management groups seemed to see their role much more as consumers than managers. They attached great importance to their close contact with patients in their day to day environment and thought that they were well placed to understand and express the impact of NHS activity (or lack of it) in human terms.

Opinions were sought from unit administrators and directors of nursing services as to the contribution that they looked to unit medical representatives to provide. The unit administrators and directors of nursing services said that they wanted unit medical representatives to be prepared to support corporate decisions; to direct medical colleagues through the unit management group rather than the district management team; to keep the unit management group informed of issues in clinical services that were likely to affect the unit's resources; and to give advice on what actions were likely to be accepted or opposed by medical staff. Unit administrators and directors of nursing services want unit medical representatives to do more than protect and defend clinical interests and to give medical opinion. They would welcome a more active role of questioning, making the unit management group aware of clinical issues, helping to devise strategies, and "standing up to be counted" along with the rest of the unit management group. For their part, unit medical representatives are conscious of the limit to their capacity to act on behalf of doctors generally and the risks to their position if they behave in a manner that alienates other doctors. They see their power to influence others as arising from their personal credibility and from the conspicuous good sense of the decisions and actions that they promote.

\section{What are the satisfactions, if any?}

The unit medical representatives were asked how they felt about their experiences-the things that they had found demanding and stressful and those that had given satisfaction and a sense of the worthwhileness of the job. The list of negative factors was the longer of the two and this seems to reflect the stage of progress that unit management groups had reached, bearing in mind that interviews took place a year or so after the inception of the new arrangements. In no case did unit medical representatives receive any documented guidance on what was expected of them and they found the consequential sense of uncertainty uncomfortable. They were unclear about what reaction was expected from them to the numerous reports and other documents that they were expected to read and, despite appreciative comments about the presence at unit management group meetings of a financial specialist, most doctors found financial statements difficult to comprehend.

When asked to list the factors that they thought distinguished one unit medical representative job from another, not one doctor made reference to the size of the unit budget although this would feature near the top of any similar list completed by a unit administrator or director of nursing services.

Moreover, many unit medical representatives thought that the primary function of the group that they had joined was to implement national policies on containment of staff numbers and they did not enjoy membership of a group that had acquired the unpopular reputation of a "cutter." "Our major function is to act as hatchet men as far as jobs go." Conflicts with fellow consultants (although recognised as not to be taken personally) were stressful. Some unit medical representatives resented the way in which district officers dealt with the unit management group and complained of work put into plans and proposals that, once submitted to district for consideration, were either rejected or seemingly ignored. The rewarding aspects of the role were less specific. A few doctors commented on the sense of participation, or indeed power, which their position gave them - having information that others did not or having it sooner. Generally the satisfactions were longer in the making and many doctors expressed gratification that their group was making progress, coming to have a better comprehension of what was needed, and enjoying the teamwork. The greatest reward seemed to come when decisions had been made and then carried through, particularly where these led to a clear improvement in services provided.

\section{Qualities required by unit medical representative}

Each unit medical representative was asked what qualities he would seek if asked to recommend a successor. A great variety of insights emerged into the characteristics that an ideal unit medical representative might possess:

(1) The confidence of clinicians, probably because he is already a senior doctor actively concerned in clinical work. In all but two of the units investigated the unit management group included at least one clinician from the unit's principal specialty (or a surgeon or physician in an acute unit). At these two units one unit medical representative was an anaesthetist and the other a pathologist, each deriving considerable status with colleagues as a result of his longstanding association with the hospital.

(2) The ability to represent the views of others diplomatically and without prejudice.

(3) The ability to collaborate effectively with other members of the unit management group.

(4) The qualities of a good listener, patient, tactful, and prepared to admit mistakes.

(5) Sufficient resilience to stand up for himself, put a case clearly, and not be distracted by rebuffs. "He should be able to compromise but equally be able to be unbelievably stubborn if necessary."

(6) Some experience of health service administration and finance and awareness of the political processes entailed in getting things done.

(7) Keenness to undertake unit medical representative responsibilities and commitment to the interests of the unit.

\section{Relations between unit management groups and the clinical representative system}

If the combined efforts of the unit administrator, director of nursing services, and unit medical representative to bring decision making closer to the operational level are to be successful the unit management group must be concerned in issues of priority and efficient resource use in relation to clinical services. Many factors determine the extent to which district officers pursue a policy of active devolution of responsibility to unit level-the size of the district, the degree of centralisation practised in the past, and the disposition of chief officers are examples. In the past medical staff have frequently found it more rewarding to take their concerns to the top-that is, to district chief officers, since it is at district level that departure from regular policy might be sanctioned, financial allocations revised, or new ideas incorporated into future plans. Despite the advent of unit management many consultants still see the cogwheel machinery, or an alternative form of medical representative system, as the primary mechanism whereby they achieve action on the things that concern them. As one surgeon put it, "I don't think that in the unit you represent anybody at all. The representation of the surgeons is through the medical executive committee not through the unit." Several doctors made the point that a matter of major concern was likely to be debated in many different places and that, if strenuous efforts were being made to influence the health authority, representation would go forward through every available route both formal and informal. If a problem is special because of its urgency or complexity then exceptional measures may be justified. Sound decision making can, however, be established only if exceptional measures are confined to exceptional cases and if a recognised and well understood pattern for debate exists and has the confidence of all protagonists. In the context of devolution of decision making to unit level this must surely mean effective collaboration between the unit management group and the medical advisory machinery at unit level. At two single specialty units covered by this study the unit management group seems to be the substantial decision maker so far as matters affecting clinicians are concerned: in the absence of a strong cogwheel system consultants wishing to pursue a matter ask their unit medical representative to take it to a unit management group meeting with a view to subsequent referral to the district management team. Five other units, two of which are acute hospital units, provide examples where the combination of unit management group and clinical division or hospital staff committee seems to be regarded as the relevant decision making mechanism. In the remaining units the unit management group seems to be of less importance in matters affecting medical staff and in four of them it is actually or virtually irrelevant in such matters.

The important factor here seems to be the status of the unit medical 
representative within the medical advisory system. Is he in a position to combine the coordination of medical opinion on the one hand and liaison with the unit administrator and director of nursing services on the other? Collaboration between the unit management group and medical staff in the unit seems to work effectively where each sees the other as a productive ally. Such an arrangement draws together the two arenas of discussion, decision and influence, which exist within the unit and provides advantages to both parties. In a multispecialty unit a hospital medical staff committee may be seen by consultants as more closely representing their collective wishes for the hospital than the divisional system. In this case the link between hospital medical staff committee and unit management group, with the medical staff committee chairman acting as unit medical representative, can work to mutual advantage. Similarly, the unit management group will feel itself more strongly placed where it is able to enlist the specific commitment of the hospital medical staff committee to any proposal that it may wish to put forward to the district. As one unit medical representative said, "If we have proposals for future developments we can dream them up in the uni management group and try to sell them to the medical staff. If we are successful in selling them to the medical staff we can say to our district management team, 'We have dreamed up these developments and they are obviously first class because the medical staff are unanimously in agreement with them.",

\section{Clinicians as unit general managers}

It is widely forecast that many unit general manager positions will be occupied by doctors, some of whom inevitably will not have gained experience as unit medical representatives. What then is a doctor likely to bring to a unit general manager role that can be counted among his strengths, and where may the pitfalls lie? The frustrations that unit medical representatives have reported in the course of this research have shown how far the rhythms of the management process in a large organisation differ from those that are normal in clinical practice. Several unit medical representatives expressed scepticism about whether their unit management group served any real purpose. Many found the apparent indecisiveness or indifference of district management teams difficult to live with. General management and delegation may speed up decision making but unless decisions are to be arbitrary and dictatorial then inevitably delays will occur, plans will need to be rewritten, and much of the work undertaken by unit general managers and other managers will continue to seem unproductive. Doctors moving into a unit general manager position will need to brace themselves for a move from a more tightly ordered, scientifically rooted, and factually defined environment to one in which ambiguity and imprecision are the norm. Further difficulty concerns the interpretation and use of management information, particularly financial information. Many unit medical representatives were unfamiliar with financial statements and found them confusing. Unit general managers will be unwise if they rely on others to interpret their financial reports. They will need to be able to read them for themselves, challenge their contents, and perhaps demand more when they are incomplete or misleading.

Perhaps the greatest barrier to the successful establishment of the unit general manager role lies with the NHS phenomenon of professional stereotyping. Typically, officers are unaware of the extent to which their professional background colours the way in which they perceive their work setting. Differences exist in the values and thought processes that individuals apply to the issues that confront them. In the past these have been an obstacle to multiprofessional teams seeking to achieve consensus. In future, from whichever source a unit general manager may be drawn, his challenge will be to shed the professional mantie that he has until recently borne, to gain new perspectives that transcend the standpoint of any one profession. Colleagues may prove unhelpful. A unit general manager is likely to find that others within the unit will, perhaps unconsciously, push him back towards familiar and more comfortable patterns of thought and action.

The doctor who becomes unit general manager will need to adapt to the relatively unfamiliar culture of management, but the medical culture in which he is rooted confers considerable advantage. Unlike a manager from another profession, a doctor is familiar with

the diversity of clinical conditions that may be encountered and the options for treatment. This understanding provides the foundation for judgment on matters of priority and improvements in efficiency in service provision. A general practitioner who was an enthusiastic advocate of doctors as managers said, "Given the right doctor he is a better manager than the administrator because of his medical overview." This goes further than simply understanding the terminology. It is a matter of a language and intellectual framework shared between the doctor/manager and fellow clinicians, common ground with colleagues, which is unavailable to anyone of a nonclinical profession. Another strength on which many doctors moving into general management can draw will be a substantial period of participation with the institutions and services that it is the business of the unit to provide. Administrators and others are frequently relatively short term incumbents of senior management positions whereas a consultant may have knowledge of the unit's history, traditions, and personalities. Looking to the future, he may also have a longer term commitment to the institutions and people who are encompassed by the unit. At the same time the professional roots that he will retain outside the management system give him a certain independence of status as a potential unit general manager. He may bring fresh thinking to the way in which the unit is managed and this may mean that new ideas can be introduced. He may feel less inhibited than the career administrator and able to take a strong line in pursuance of the beliefs that he has acquired. A conflict of view with the district general manager or health authority can be pursued by a doctor with vigour in the knowledge that, if the worst comes to the worst, the option of a return to full time clinical duties is probably available to him.

\section{THIRTY YEARS AGO}

\section{The Cost of Subscriptions}

SIR, - With the present efforts of the B.M.A. to obtain income-tax relief for subscriptions to professional societies and journals, and with the recent announcements of an increase in some of these subscriptions, it is opportune to consider these mounting expenses against the present-day background. As all of us know, the cost of living continues to rise, and as one group of people (ourselves included) get a salary increase so others follow, and we must see that in our turn as employers our officers and other employees have a proper salary. There is, therefore, no doubt that the rise in the cost of professional societies is unavoidable, for not only wages and salaries but also the cost of fuel, paper, etc., have risen. Can this increase in costs only be met by a rise in subscriptions? Furthermore, will a rise in subscription ultimately benefit the society concerned?

As a member of the public health department of a local authority, with duties mainly concerned with occupational health, I have paid this year (1954-5) the following subscriptions:

SOCIETIES

British Medical Association Royal Society of Medicine Royal Sanitary Institute Society of Medical Officers of Health Association of Industrial Medical Officers

JOURNALS:

Lancet

Medical Office

Brit. F. Indust. Med

Brit.F. Social Med.

Bull. Hyg. (reprints)

OTHER SUBSCRIPTIONS:

Lewis's library (3 vols.)

Medical Defence Union

Royal Medical Benevolent Fund

Public Health Defence Trust

$$
\text { Total: }
$$

